Publ. RIMS, Kyoto Univ.

18 (1982), 119-130

\title{
Order Properties of a Class of Tensor Algebras
}

By

\author{
Julio AlCÁntARA*
}

\begin{abstract}
By means of an auxiliary coarser topology we study certain order properties of inductive tensor algebras over nuclear $L F$-spaces.
\end{abstract}

\section{$\S 1$. Introduction, Notations and Statement of Results}

A locally convex ${ }^{*}$-algebra $\mathscr{A}[\mathscr{g}]$ is a locally convex space equipped with a separately continuous multiplication

$$
x: \mathcal{A}[\mathscr{g}] \times \mathcal{A}[\mathscr{g}] \rightarrow \mathscr{A}[\mathscr{g}]
$$

and a continuous involution

$$
*: \mathcal{A}[\mathcal{g}] \rightarrow \mathcal{A}[\mathcal{g}] \text {. }
$$

The separate continuity of the multiplication means that for all $g \in \mathscr{A}$ the maps $f_{\rightsquigarrow \rightarrow g} \times f$ and $f \rightsquigarrow f \times g$ from $\mathcal{A}[g]$ to $\mathcal{A}[g]$ are continuous. It is also assumed that $\mathcal{A}$ has a unit satisfying $\mathbb{1}=\mathbb{1}^{*}$.

An order structure is introduced on $\mathcal{A}_{h}=\left\{f \in \mathcal{A}: f=f^{*}\right\}$, the real subspace of hermitian elements of $\mathcal{A}$, by defining the cone of positive elements, $\overline{\mathscr{A}}_{+}$, to be the closure of the set

$$
\mathscr{A}_{+}=\left\{\sum_{i=1}^{n} f_{i}^{*} \times f_{i}: f_{i} \in \mathscr{A}, 1 \leq i \leq n, n \in \mathbb{N}\right\} \text {. }
$$

The cone $\overline{\mathscr{A}}_{T}$ determines a transitive and reflexive partial order on $\mathscr{A}_{h}$, and we write $f \geq g$ whenever $f-g \in \overline{\mathcal{A}}_{+}$. When this order is antisymmetric the cone $\overline{\mathscr{A}}_{+}$is called proper. Evidently $\overline{\mathscr{A}}_{+}$is proper if and only if $\overline{\mathscr{A}}_{+} \cap-\overline{\mathscr{A}}_{+}=\{0\}$.

Communicated by H. Araki, January 30, 1981.

* Faculty of Mathematics, The Open University, Walton Hall, Milton Keynes, MK7 6AA, England.

On leave from the U.P.C.H., Lima, Perú. 
If $f, g \in \mathcal{A}_{h}$ the order interval $[f, g]$ is defined by

$$
[f, g]=\left\{k \in \mathcal{A}_{h}: f \leq k \leq g\right\} .
$$

The subset $\left\{\mu f: \mu \in \boldsymbol{R}^{+}, f \in \overline{\mathscr{A}}_{+}\right\}$of $\overline{\mathscr{A}}_{+}$is said to be an extremal ray if $[0, f]=\{\lambda f: \lambda \in[0,1]\}$.

To $\overline{\mathscr{A}}_{+}$we associate the dual cone of positive functionals

$$
\mathscr{A}_{+}^{\prime}=\left\{T \in \mathcal{A}[g]^{\prime}: T(f) \geq 0 \quad \forall f \in \overline{\mathcal{A}}_{+}\right\} .
$$

A positive functional $T$ will be called strictly positive if

$$
T(f)>0 \quad \forall f \in \overline{\mathscr{A}}_{+}, \quad f \neq 0 .
$$

The algebras to be dealt with in this paper are the so called BUalgebras [1,3]. Given a complex nuclear $L F$-space (strict inductive limit of Fréchet spaces) $E$, the BU-algebra over $E$ is the locally convex direct sum tvs

$$
\underline{E}=\bigoplus_{n=0}^{\infty} \bar{\otimes}^{n} E
$$

where $n=0$ corresponds to $C$ by convention and $\bar{\otimes}$ indicates the completion of the tensor product in the inductive tensor product topology ([12], p. 96 and p. 119, Exercise 22). The product with respect to which $E$ is an algebra follows from its graded structure:

$$
\text { if } \underline{f}=\left(f_{0}, f_{1}, \cdots, f_{r}, 0,0, \cdots\right), \underline{g}=\left(g_{0}, g_{1}, \cdots, g_{s}, 0,0, \cdots\right) \in \underline{E},
$$

then

$$
\underline{f} \times \underline{\underline{g}}=\left(f_{0} g_{0}, f_{0} g_{1}+f_{1} g_{0}, \cdots, \sum_{i+j=p} f_{i} \otimes g_{j}, \cdots, f_{r} \otimes g_{s}, 0,0, \cdots\right) .
$$

It is further assumed that a continuous involution, ${ }^{*}$, is defined on $E$. In an obvious way this extends linearly to an involution

$$
\underline{f}_{m \rightarrow} \underline{f}^{*} \text { on } \underline{E}, \text { with }(\lambda \underline{f} \times \underline{g})^{*}=\bar{\lambda} \underline{g}^{*} \times \underline{f}^{*} \text {. }
$$

In $[1,3,4]$ BU-algebras have been used to formulate some physical theories that deal with infinite systems.

In addition to their role in applications, BU-algebras are important because of the following structure theorem [2]. Every $I^{*}$-algebra is isomorphic to the quotient of a BU-algebra by a complemented *-invariant positive ideal. An $I^{*}$-algebra $\mathcal{A}[\mathcal{g}]$ is a locally convex complex unital *-algebra with a proper incomplete cone $\mathcal{A}_{+}$and a nuclear $L F$-topology 
g. Such algebras are discussed in $[1,3]$.

The principal results of this paper are that, for any BU-algebra $\underline{E}$. (a) the positive cone $\underline{E}_{+}$of $E$ is given explicitly by the set of convergent series:

$$
\underline{E}_{4}=\left\{\sum_{i=1}^{\infty} f_{i}^{*} \times f_{i}: f_{i} \in \underline{E}\right)
$$

(b) the ordcr intervals of $\underline{E}$ are compact and $\underline{E}_{+}$is the closed convex hull of its extremal rays;

(c) E has a strictly positive functional if and only if E has a continuous norm.

This paper is organized as follows. In Section 2 we prove some preliminary lemmas which we leel to be of some independent interest. The main results are proven in Section 3, and we finish with a list of unsolved problems.

\section{§2. Preliminary Results}

Before proving our first lemma we need to introduce the following definition.

Definition. A seminorm $P$ on a direct sum $\bigoplus_{n=0}^{\infty} E_{n}$ will be called graded if it is of the form $P=\sum_{n \geq 0} p_{n}$, where $p_{n}$ is a seminorm on $E_{n}$. A locally convex topology on a tensor algebra will be called graded if it has a generating family of seminorms that are graded.

In general the multiplication in tensor algebras is not jointly continuous. For example the multiplication in a BU-algebra is jointly continuous if and only if $E$ is an $L B$-space ([1], Corollary 1.13 or [3], Proposition 1.7). If $E$ is a complex nuclear space with a continuous involution and $\hat{\otimes}^{n} E$ is the $n$-fold completed projective tensor product, the lemma below gives some properties of the finest graded topology $g_{\infty}$ on $\underset{\sim}{E} \equiv \bigoplus_{n=0}^{\infty} \widehat{\otimes}^{n} E$, coarser than the original topolngy, for which the multiplication is jointly continuous, i.e., for which the map

$$
\times: \underset{\sim}{E}\left[\mathscr{g}_{\infty}\right] \times \underset{\sim}{E}\left[\mathscr{g}_{\infty}\right] \rightarrow \underset{\sim}{E}\left[\mathscr{G}_{\infty}\right]
$$


is continuous.

Lemma 1. Let $E$ be a complex nuclear space with a continuous involution. Then the finest graded topology $g_{\infty}$ on $\underset{\sim}{E}$, coarser than the original one, for which the multiplication is jointly continuous, has the following properties:

(i) the involution $*: \underset{\sim}{E}\left[\mathscr{g}_{\infty}\right] \rightarrow \underset{\sim}{\mathbb{E}}\left[\mathcal{g}_{\infty}\right]$ is continuous and on $\bigoplus_{n=0}^{N} \widehat{\otimes}^{n} E, N$ finite, $g_{\infty}$ induces the original topology;

(ii) $\underset{\sim}{E}\left[g_{\infty}\right]$ is a nuclear space;

(iii) the cone $\underset{\sim}{\bar{E}_{+}}$is $\mathscr{g}_{\infty}$-normal;

(iv) the topology defined by the seminorms $f_{\mathrm{m} \rightarrow} T\left(f^{*} \times f\right)^{1,2}$, for all $g_{\infty}$-continuous positive functionals $T$. is equal to $g_{\infty}$.

Proof. If $\left(p_{\hat{o}}\right)_{\partial \in d}$ is a generating family of seminorms for $E$ it can be shown that

$$
P_{\gamma, \delta}=\sum_{n \geq 0} \gamma_{n}\left(p_{\delta} \otimes_{\pi} \cdots \otimes_{\pi} p_{\delta}\right)
$$

where $\underset{\sim}{\gamma}=\left(\gamma_{n}\right)_{n \geq 0}$ varies over all sequences of non-negative numbers and $\delta \in A$, is a generating family of seminorms for $\underset{\sim}{E}\left[\mathscr{g}_{\infty}\right]$. The assertions in (i) follow immediately from this.

To prove property (ii) it is convenient to replace the seminorms $P_{r, \delta}$ by the equivalent family of seminorms

$$
P_{z, \delta}^{\prime}=\left[\sum_{n \geq 0} \gamma_{n}\left(p_{\delta} \otimes_{\pi} \cdots \otimes_{\pi} p_{\delta}\right)^{2}\right]^{1 / 2}
$$

and take the $\left(p_{\delta}\right)_{\delta \in d}$ to be Hilbertian seminorms. This is always possible because $E$ is nuclear. By nuclearity, for any $p_{\delta}$, there exists a $p_{\omega}(\delta, \omega$ $\in \Delta)$ such that $p_{\omega}$ dominates $p_{\delta}$ and the canonical injection $i_{\omega o}$ from $\mathscr{H}_{\omega}$, the Hilbert space completion of $E / p_{\omega}^{-1}(0)$, into $\mathcal{H}_{\delta}$ is Hilbert-Schmidt. Without loss of generality $p_{\omega}$ can be chosen so that the Hilbert-Schmidt norm of $i_{\omega \delta}$ is less than 1 . Then it is not difficult to show that the natural injection $i_{\tau, \omega \delta}$ from $\mathcal{H}_{\mathcal{\tau}, \omega}$, the Hilbert space completion of $\underset{\sim}{E} / P_{\tilde{\gamma}, \omega}^{\prime-1}(0)$, into $\mathcal{H}_{\mathcal{I}, \delta}$ is also Hilbert-Schmidt.

Property (iii) follows from the nuclearity of $E$ by using the seminorms $P_{\tau, \delta}^{\prime \prime}=\sum_{n 20} \gamma_{n} p_{\delta} \otimes_{\varepsilon} \cdots \otimes_{\varepsilon} p_{\delta}$ in [6], Satz 1 .

Finally the nuclearity of $\underset{\sim}{E}\left[g_{\infty}\right]$ and the $g_{\infty}$-normality of $\underset{\sim}{\bar{E}_{+}}$imply 
that for every $g_{\infty}$-continuous seminorm $P$ there is a summable sequence of positive numbers $\left(\lambda_{n}\right)_{n \geq 1}$ and a $g_{\infty}$-equicontinuous sequence of positive functionals $\left(T_{n}\right)_{n \geq 1}$ such that

$$
P(f)^{2} \leq \sum_{n \geq 1} \lambda_{n}\left|T_{n}(f)\right|^{2}
$$

([10], Théorème 3). By the Cauchy-Schwarz inequality for positive functionals,

$$
\sum_{n \geq 1} \gamma_{n}\left|T_{n}(f)\right|^{2} \leq \sum_{n \geq 1} \lambda_{n} T_{n}(\mathbb{1}) T_{n}\left(f^{*} \times f\right) .
$$

Therefore $T \equiv \sum_{n \geq 1} \lambda_{n} T_{n}$ (1) $T_{n}$ is a $\mathscr{G}_{\infty}$-continuous positive functional such that $P(f)^{2} \leq T\left(f^{*} \times f\right)$. Property (iv) follows from this inequality, the $\mathcal{F}_{\infty}$-continuity of the involution and the $\dot{y}_{\infty}$-joint continuity of the multiplication.

积

The $\mathscr{y}_{\infty}$ topology of a BU-algebra $\underline{E}$ also satisfies Lemma 1, because it has $\left\{P_{z, 0}\right\}$ as a generating family of seminorms. It is also worth mentioning that if $g$ is the original topology on $E$ and $T$ is a $g$-continuous positive functional, then the seminorm $f_{\rightsquigarrow \rightarrow} T\left(f^{*} \times f\right)^{1 / 2}$ is $g$ continuous. But the analog of part (iv) of Lemma 1 for $g$ holds when $E$ is a Fréchet nucler space if and only if $E$ is isomorphic to a closed subspace of $s$, the Fréchet space of rapidly decreasing sequences (Yngvason [17], Satz 4.8 and private communication); when $E$ is a nuclear $L F$-space such that $E \otimes_{6} E \neq E \otimes_{\pi} E$ it never holds ([3], Proposition 2.8).

Our next lemma deals with the problem of extension of positive functionals in tensor algebras.

Lemma 2. (i) Let E be a complex nuclear spacc with a continuous involution and, for finite $N$, suppose that $T=\left(T_{0}, T_{1}, \cdots, T_{2 N}\right.$, $0,0, \cdots)$ is a continuous linear functional on $\underset{\sim}{E}$ which is positive on $\bigoplus_{n=0}^{2 N} \widehat{\otimes}^{n} E$. Then there is a $g_{\infty}$-continuous positive functional $S$ such that for all $\varepsilon>0$ one can find a sequence of positive type $\left(\alpha_{n}\right)_{n \geq 0}$, with $\max _{0 \leq n \leq 2 N}\left|\alpha_{n}\right|<\varepsilon$, so that $T+S_{\left(\alpha_{n}\right)}$ is a positive functional. Here $S_{\left(\alpha_{n}\right)}$ $\equiv\left(\alpha_{0} S_{0}, \alpha_{1} S_{1}, \cdots, \alpha_{n} S_{n}, \cdots\right)$.

(ii) Let $E$ be a complex nuclear space with a coniinuous involution and $G$ a closed *-invariant subspace of $E$. If $T$ is a $\mathscr{g}_{\infty}$ - 
continuous positive functional on $G$, then it has a positive extension to $\underset{\sim}{E}$.

Proof. (i) This will follow as in [17], pp. 17-18, Lemma, if there are a continuous seminorm $P$ and a positive functional $S$ such that $\left|T\left(f^{*} \times g\right)\right| \leq P(f) P(g)$ and $P(f)^{2} \leq S\left(f^{*} \times f\right)$. Since $T$ is $g_{\infty}$-continuous and $\underset{\sim}{\bar{E}_{+}}$is $\mathscr{g}_{\infty}$-normal (Lemma 1 (iii)), there are $\mathscr{g}_{\infty}$-continuous positive functionals $T_{1}, T_{2}$ such that $T=T_{1}-T_{2}$ ([12], p. 220, Corollary 3). Using in part the Cauchy-Schwarz inequality for positive functionals we get $\left|T\left(f^{*} \times g\right)\right| \leq P(f) P(g)$, where $P(f) \equiv T_{1}\left(f^{*} \times f\right)^{1 / 2}+T_{2}\left(f^{*} \times f\right)^{1 / 2}$ is a $g_{\infty}$-continuous seminorm (Lemma 1 (iv)). Finally by Lemma 1 (iv) there is a $g_{\infty}$-continuous positive functional $S$ such that $P(f)^{2}$ $\leq S\left(f^{*} \times f\right)$.

(ii) This is proven in [18], Theorem 8 , when $T$ is dominated by a seminorm of the form $\sum_{n \geq 0} r_{n} p \otimes_{\pi} \cdots \otimes_{\pi} p$, where $p$ is a *-invariant Hilbertian norm on $E$. When $E$ does not have a continuous norm a similar proof goes through if we work with the spaces $E / p^{-1}(0)$ and $G / p^{-1}(0)$ instead of $E$ and $G$, respectively.

Our last lemma is partly a generalization of the following result of Schmüdgen ([13], Section 3, Lemma 2): Let $E$ be a complex Fréchet space with a continuous involution $f m \rightarrow f^{*}$. Then the closure of the cone

$$
(E \otimes E)_{+}=\left\{\sum_{i=1}^{n} f_{i}^{*} \otimes f_{i}: f_{i} \in E, 1 \leq i \leq n, n \in N\right\}
$$

in $E \widehat{\otimes}_{\varepsilon} E$. the completion of the tensor product in the injective topology, is

$$
\left\{\sum_{i=1}^{\infty} f_{i}^{*} \otimes f_{i}: f_{i} \in E\right\}
$$

Lemma 3. Let $E$ be a complex nuclear LF-space with a continuous involution $f_{m \rightarrow} f^{*}$. Then the closure of the cone

$$
(E \otimes E)_{+}=\left\{\sum_{i=1}^{n} f_{i}^{*} \otimes f_{i}: f_{i} \in E, 1 \leq i \leq n, n \in \mathbb{N}\right\}
$$


in $E \bar{\otimes} E$ is

$$
\left\{\sum_{i=1}^{\infty} f_{i}^{*} \otimes f_{i}: f_{i} \in E\right\}
$$

Proof: By [15], p. 134, Exercise 13.4, $E$ has a sequence of defini-

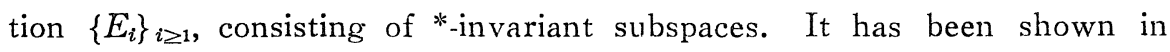
[1], Proposition A. 49 , that $E \bar{\otimes} E$ is a nuclear $L F$-space with a sequence of definition $\left\{E_{i} \widehat{\otimes} E_{i}\right\}_{i \geq 1}$. (As $E_{i}$ is nuclear, we have omitted the subscript $\varepsilon$ in $E_{i} \widehat{\otimes}_{\varepsilon} E_{i}$.

We are going to show that

$$
\overline{(E \otimes E)_{+}}=\bigcup_{i=1}^{\infty} \overline{\left(E_{i} \otimes E_{i}\right)_{+}}
$$

and the result will then follow from Schmüdgen's Lemma. By [16], Theorem 2.15,

$$
\overline{(E \otimes E)_{\mathrm{r}}}=\left\{h \in E \bar{\otimes} E: T(h) \geq 0, \forall T \in(E \otimes E)_{+}^{\prime}\right\} .
$$

It is straightforward to show that all the extremal rays of the dual cone $(E \otimes E)_{+}^{\prime}$ are of the form $l^{*} \otimes l$, where $l \in E^{\prime}$ and $l^{*}(f) \equiv \overline{l\left(f^{*}\right)}$. Since $E \bar{\otimes} E=\bigcup_{j=1}^{\infty}\left(E_{i} \widehat{\otimes} E_{i}\right)$, every element $u$ of $E \bar{\otimes} E$ has a representation of the form

$$
u=\sum_{j=1}^{\infty} \lambda_{j} f_{j} \otimes g_{j}
$$

where $\sum_{j=1}^{\infty}\left|\lambda_{j}\right|<+\infty$ and $\left\{f_{j}\right\}_{j \geq 1},\left\{g_{j}\right\}_{j \geq 1}$ are null sequences in $E$ ([12], p. 94, Theorem 6.4). A simple polarization argument then shows that the cone $\overline{(E \otimes E)}+$ is generating, i.e., the smallest subspace containing it is $E \bar{\otimes} E$. Consequently the order intervals associated to the dual cone $(E \otimes E)_{+}^{\prime}$ are bounded in the $\sigma\left((E \bar{\otimes} E)^{\prime}, E \bar{\otimes} E\right)$ topology and therefore compact, because the strong dual $(E \bar{\otimes} E)^{\prime}$ of $E \bar{\otimes} E$ is Montel ([1], Proposition 1.2 or [3], Lemma 1.2). Since $(E \bar{\otimes} E)^{\prime}$ is dual nuclear and complete ([1], Proposition 1.2 or [3], Lemma 1.2), the compactness of its order intervals implies that $(E \otimes E)_{+}^{\prime}$ is the closed convex hull of its extremal rays ([14], Théorème 1, Corollaire), because $(E \otimes E)_{+}^{\prime}$ is proper $(\overline{(E \otimes E)}+$ is generating) and closed. Therefore

$$
\overline{(E \otimes E)}_{+}=\left\{h \in E \bar{\otimes} E:(l * \otimes l)(h) \geq 0, \forall l \in E^{\prime}\right\} .
$$


Similarly

$$
{\overline{\left(E_{i} \otimes E_{i}\right)_{+}}}=\left\{k \in E_{i} \widehat{\otimes} E_{i}:\left(l_{i}^{*} \otimes l_{i}\right)(k) \geq 0, \quad \forall l_{i} \in E_{i}^{\prime}\right\} .
$$

By the Hahn-Banach extension theorem we then get

$$
\overline{(E \otimes E)}+\cap\left(E_{i} \widehat{\otimes} E_{i}\right)=\overline{\left(E_{i} \otimes E_{i}\right)}+
$$

which finishes the proof of the lemma.

\section{§ 3. Main Results}

Theorem 1. Let $E[g]$ be a BU-algebra. Then

$$
\underline{\bar{E}}^{+}=\left\{\sum_{i=1}^{\infty} f_{i}^{*} \times f_{i}: f_{i} \in \underline{E}\right\} .
$$

Proof. First we will prove the result when $E$ is a Fréchet nuclear space. Note that in this case $E$ is isomorphic to $\underset{\sim}{E}$ ([5], chapter 1, p. 74). Since the algebra $\bigoplus_{n=0}^{\infty} \bigotimes_{n}^{n} E$ is dense in $\underset{\sim}{E}$, it follows that $\underset{\infty}{\bar{E}_{+}}$ $=\bar{K}_{+}$, where $K_{+}$is the positive cone of the algebra $\bigoplus_{n=0}^{\infty} \bigotimes_{\pi}^{n} E$. Next it will be shown that the closure of $K_{+}$is equal to its sequential closure. If $F_{i} \equiv \bigoplus_{n=0}^{2 i} \widehat{\otimes}^{n} E$, then $\underset{\sim}{E}=\bigcup_{i=1}^{\infty} F_{i}$ and therefore $\bar{K}_{+}=\bigcup_{i=1}^{\infty}\left(\bar{K}_{+} \cap F_{i}\right)$. Part (i) of Lemma 2 implies that the set of positive functionals on $F_{i}$ that have a positive extension to $\underset{\sim}{E}$ is dense in the set of positive functionals on $F_{i}$. Consequently $\bar{K}_{+} \cap F_{i}=\bar{K}_{+} \cap F_{i}$ (cf. [16], Theorem 2.15). This completes the proof that the closure of $K_{+}$is equal to its sequential closure because $F_{i}$ is metrizable if $i$ is finite.

We now show that if $\left\{u_{n}=\sum_{i=1}^{r_{n}} f_{i, n}^{*} \times f_{i, n}: n \in N\right\}$ is a convergent sequence in $K_{+}$, then the set $\left\{v_{n}=\sum_{i=1}^{r_{n}} f_{i, n}^{*} \otimes f_{i, n}: n \in N\right\}$ is bounded in $\underset{\sim}{E}[g] \bar{\otimes} \underset{\sim}{E}[g]$. If $p$ is a $g_{\infty}$-continuous seminorm then

$$
\left(p \otimes_{\pi} p\right)\left(v_{n}\right) \leq \sum_{i=1}^{r_{n}} p\left(f_{i, n}\right)^{2} \leq \sum_{i=1}^{r_{n}} T\left(f_{i, n}^{*} \times f_{i, n}\right)=T\left(u_{n}\right)
$$

by part (iv) of Lemma 1. Therefore $\left(v_{n}\right)_{n \geq 1}$ is bounded in $\underset{\sim}{E}\left[\mathscr{g}_{\infty}\right] \otimes_{\pi}$ $\underset{\sim}{E}\left[g_{\infty}\right]$. Since there is a finite $i$ such that $\left(v_{n}\right)_{n \geq 1} \subset F_{i} \otimes F_{i}$, Lemma 1 (i) implies that $\left(v_{n}\right)_{n \geq 1}$ is bounded in $F_{i} \bigotimes_{\pi} F_{i}$. This set is also bounded in $\underset{\sim}{E}[g] \bar{\otimes} \underset{\sim}{E}[\mathcal{g}]$, because this space is the strict inductive limit of $\left\{F_{i}\right.$ $\left.\widehat{\otimes} F_{i}\right\}_{i \geq 1}$ (cf. [1], Propositions A. 49 and A.50). By the nuclearity of 
$\underset{\sim}{E}[g] \bar{\otimes} \underset{\sim}{E}[g] \quad\left([1]\right.$, Proposition A. 49), $\left(v_{n}\right)_{n \geq 1}$ has a convergent subsequence $\left(w_{n}\right)_{n \geq 1}\left([12]\right.$, p. 101, Corollary 2) and by Lemma 3, $\lim _{n} w_{n}$ $=\sum_{i=1}^{\infty} f_{i}^{*} \otimes f_{i}$. The separate continuity of the multiplication in $\underset{\sim}{E}[\mathscr{g}] \mathrm{im}$ plies that the map $M: \underset{\sim}{E}[g] \bar{\otimes} \underset{\sim}{E}[g] \rightarrow \underset{\sim}{E}[g] ; M(f \otimes g) \equiv f \times g$, is continuous ([1], Proposition 1.10 or [3], Proposition 1.7). Consequently

$$
\lim _{n} u_{n}=\lim _{n} M\left(w_{n}\right)=M\left(\lim _{n} w_{n}\right)=\sum_{i=1}^{\infty} f_{i}^{*} \times f_{i} .
$$

This finishes the proof of the Theorem when $E$ is a nuclear Frechet space.

Before dealing with the last part of the proof, note that by Lemma 2 (i), for any complex nuclear space $E$ with a continuous involution, the $g_{\infty}$-closure of $\underset{\sim}{E}, \underset{\sim}{\bar{E}_{+}^{g}}$, is equal to $\underset{\sim}{\bar{E}_{+}}$. The same conclusion holds for

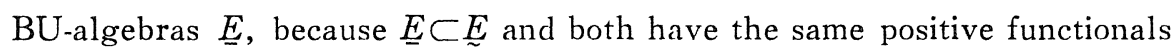
([3], Proposition 2.8) and the same $g_{\infty}$-continuous positive functionals (see first remark after Lemma 1).

To conclude the proof of the theorem assume now that $E$ is a

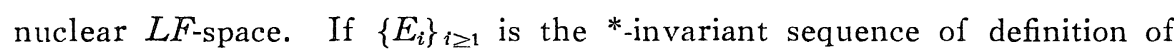
$E$ introduded in the proof of Lemma 3, then $E=\bigcup_{i=1}^{\infty} \underset{\sim}{E_{i}}$ (cf. [1], Propositions A. 49 and A. 50) and therefore $\underline{E}_{+}=\bigcup_{i=1}^{\infty}\left(\underline{E}_{+} \cap \bigcap_{i=1} \underline{E}_{i}\right)$. By Lemma 2

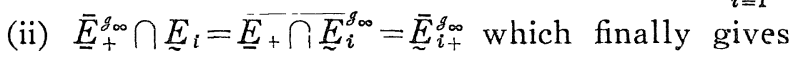

$$
\underline{E}_{+}=\bar{E}_{+}^{g_{\infty}}=\bigcup_{i=1}^{\infty}\left(\bar{E}_{+}^{g_{\infty}} \cap \underline{\sim}_{i}\right)=\bigcup_{i=1}^{\infty}{\underset{\sim}{E_{i+}}}_{g_{\infty}}=\bigcup_{i=1}^{\infty}{\underset{\sim}{E}}_{i+}
$$

finishing the proof of the theorem because $\underline{E}_{i}$ is a nuclear Fréchet space.

图

Theorem 2. Let $\underline{E}[\mathcal{g}]$ be a BU-algebra. Then its order interrals are compact, and $\underline{\underline{E}}_{+}$is the closed convex hull of its extremal rays.

Proof. Since $E[g]$ is a Montel space ([1], Proposition 1.2 or [3], Lemma 1.2) and the order intervals are closed, their compactness will follow from boundedness. Let $\left\{E_{i}\right\}_{i \geq 1}$ be a *-invariant sequence of definition of $E$ and $G_{i} \equiv \bigoplus_{n=0}^{2 i} \widehat{\otimes}^{n} E_{i}$. As $\bar{\otimes}^{n} E$ and $\widehat{\otimes}^{n} E$ induce the original topology on $\widehat{\otimes}^{n} E_{i}$ ([1], Proposition A. 49 and [8], p. 119), the topology $g_{\infty}$ induces the original topology on $G_{i}$ and therefore by Lemma 1 (iii) 
and the first remark after Lemma $1, \underline{E}_{+} \cap G_{i}$ is normal. Consequently if every order interval of $E$ is contained in a subspace $G_{i}$, they will be bounded ([12], p. 216, Corollary). Let $f \in \underline{E}_{+}$and $0 \leq g \leq f$. Since $\underline{E}$ $=\bigcup_{i=1}^{\infty} G_{i}$ (cf. [1], Propositions A. 49 and A. 50), there is an $i$ such that $f \in G_{i}$. It will be shown that $g \in G_{i}$. If $l$ is a hermitian linear functional on $G_{i}$, then there exist positive linear functionals $T_{1}, T_{2}$ on $G_{i}$ such that $l=T_{1}-T_{2}$ ([12], p. 220, Corollary 3). If $\widetilde{T}_{1}$ and $\widetilde{T}_{2}$ are the extensions of $T_{1}$ and $T_{2}$ to $\underset{\sim}{E}$, taking the value zero on $\bigoplus_{n=2 i+1}^{\infty} \widehat{\otimes}^{n} E_{i}$, part (i) of Lemma 2 implies that there are positive functionals $S_{T_{1}}$ and $S_{T_{2}}$ on $\underset{\sim}{E_{i}}$, with $S_{T_{j}}(f)<1(j=1,2)$, such that $\widetilde{T}_{j}+S_{T_{j}}(j=1,2)$ are positive. By part (ii) of Lemma $2, \widetilde{T}_{j}+S_{T_{j}}$ and $S_{T_{j}}(j=1,2)$ have positive extensions $V_{T_{j}}$ and $U_{T_{j}}(j=1,2)$, respectively, to $\underline{E}$, because $\underline{E} \subset \underset{\sim}{E}$. If $\tilde{l}(g)$ $\equiv V_{T_{1}}(g)-V_{T_{2}}(g)+U_{T_{2}}(g)-U_{T_{1}}(g)$, we are going to prove that $l_{w \rightarrow}|\tilde{l}(g)|$ is a continuous seminorm on the hermitian part $G_{i, h}^{\prime}$ of $G_{i}^{\prime}$. The reflexivity of $G_{i}$ will then imply that $g \in G_{i}$. Since $G_{i, h}^{\prime}$ is bornological (cf. [1], Proposition 1.2 or [3], Lemma 1.2) we need to verify that if $B$ is a bounded subset of $G_{i, h}^{\prime}$, then

$$
\sup _{l \in B}|\tilde{l}(g)|<+\infty .
$$

Now as $G_{i}$ is barreled, $B$ must be equicontinuous ([12], p. 127, Corollary). By the normality of the cone $\underline{E}_{+} \cap G_{i}$, there is an equicontinuous set of positive functionals $C$ in $G_{i, n}^{\prime}$ such that $B \subset C-C$ ([12], p. 220, Corollary 1). Therefore

$$
\begin{aligned}
\sup _{l \in B}|\tilde{l}(g)| & \leq \sup _{l \in B}\left(V_{T_{1}}(g)+V_{T_{2}}(g)+U_{T_{1}}(g)+U_{T_{2}}(g)\right) \\
& \leq \sup _{T_{1}, T_{2} \in C}\left(T_{1}(f)+T_{2}(f)+2 S_{T_{1}}(f)+2 S_{T_{2}}(f)\right) \\
& \leq 4+\sup _{T \in C} T(f)<+\infty,
\end{aligned}
$$

which finishes the proof of the first part of the Theorem.

The last part of the theorem follows from [14], Théorème 1, Corollaire, because $E[g]$ is dual nuclear and complete ([1], Proposition 1.2 or [3], Lemma 1.2), the order intervals are compact and $\underline{E}_{+}$is proper (Lemma 1 (iii), first remark after Lemma 1 and [12], p. 216, Corollary 1) and closed. 
Theorem 3. A $B C^{\top}$-algebra E has a strictly positive functional if and only if $E$ has a continuous norm,

Proof. If $p$ is a continuous norm on $E$, then $P=\sum_{n \geq 0}^{\infty} p \otimes_{\pi} \cdots \otimes_{\pi} p$ is a $g_{\infty}$-continuous norm on $E$. By part (iv) of Lemma 1 there is a $\mathscr{g}_{\infty}$ continuous positive functional $T$ such that

$$
P(f)^{2} \leq T\left(f^{*} \times f\right), \quad \forall f \in \underline{E} .
$$

This inequality and Theorem 1 imply that $T$ is strictly positive.

To prove the reverse implication note that if $T$ is a strictly positive functional, then $f_{m \rightarrow} T\left(f^{*} \times f\right)^{1 / 2}$ is a continuous norm on $\underline{E}$, as $\underline{E}$, is barreled ([9], Theorem 4.1). Obviously $E$ also has a continuous norm.

For examples of nuclear Fréchet spaces without a continuous norm see [11], Theorem 2.

We finish this paper with a short list of unsolved problems.

(1) Is it true that $\underset{E}{E} \underset{\sim}{E}$ (equality as vector spaces only), for every nuclear LF-space E? Do they have the same bounded sets?

(2) Characterize explicitly the extremal rays of BU-algebras.

(3) A subspace I of a locally convex* *algebra $A[g]$ will be called state-related if

$$
I=\bigcap\left\{K(T): T \in \mathcal{A}_{+}^{\prime} \text { and } K(T) \supset I\right\}
$$

where $K(T)$ is the kernel of $T$. Characterize the subspaces of $B U$ algebras that are state-related. In particular is the Wightman kernel of Quantum Field Theory ([7]) a state-related subspace?

\section{Acknowledgements}

I would like to thank the Open University for financial support, the U.P.C.H. (Lima-Perù) for an extended leave of absence, Dr. D. A. Dubin for reading the manuscript, and Drs. G. Hofmann, K. Schmüdgen, and J. Yngvason for making available to me copies of their work. I would also like to thank Dr. J. Yngvason for pointing out two errors in an earlier version of the paper, for bringing to our attention Lemma 
2 (i) and constructive criticism.

\section{References}

[1] Alcántara, J., $I^{*}$-Algebras and their Applications, Ph. D. Dissertation, The Open University, July, 1979.

[2] - A structure Theorem for a Class of Locally Convex *-Algebras, (in preparation).

[3] Alcántara, J. and Dubin, D., I*-Algebras and their Applications, Publ. RIMS, Kyoto Univ., 17 (1981), 179-199.

[4] - Current Commutation Relations as States on an I*-Algebra, Reports. Math. Phys., (to appear).

[5] Grothendieck, A., Produits Tensoriels Topologiques et Espaces Nucléaires, Mem. Amer. Math. Soc., 16 (1955).

[6] Hofmann, G., Ein hinreichendes Kriterium für die Normalität von Kegeln in Tensoralgebren und einige Anwendungen, Wiss. Z. Karl-Marx-Univ. Leipzig, Math. Naturwiss. R., 27 (1978), 261-269.

[7] Hofmann, G. and Lassner, G., Existence Proofs for Wightman-Type Functionals, Wiss. Z. Karl-Marx-Univ. Leipzig, Math.-Naturwiss. R., 24 (1975), 535-543.

[8] Jarchow, H., Nuclear Locally Convex Spaces, Lecture Notes No. 13, University of Maryland, 1976.

[9] Lassner, G., Topological Algebras of Operators, Reports Math. Phys., 3 (1972), 279-293.

[10] Mokobodzki, G., Cônes Normaux et Espaces Nucléaires. Cônes Semi-Complets, Séminaire Choquet (Initiation a l'Analyse) $7^{\mathrm{e}}$ année, 1967/68, No. B. 6.

[11] Moscatelli, V. B., Fréchet Spaces without Continuous Norm and without Bases, Bull. London Math. Soc., 12 (1980), 63-66.

[12] Schaefer, H. H., Topological Vector Spaces, Springer, Berlin, 1971.

[13] Schmüdgen, K., Graded and Filtrated Topological *-Algebras. The Closure of the Positive Cone, Rev. Roumaine Math. Pures Appl., (to appear).

[14] Thomas, E., Représentations Intégrale dans les Cônes Convexes, C. R. Acad. Sci. (Paris), 286A (1978), 515-518.

[15] Tréves, F., Topological Vector Spaces, Distributions and Kernels, Academic Press, New York, 1967.

[16] Wong, Y.-C. and Ng, K.F., Partially Ordered Topological Vector Spaces, Oxford University Press, London, 1973.

[17] Yngvason, J., Über den algebraischen Formalismus in der Wightmanschen Quantenfeldtheorie, Habilitationsschrift, Göttingen, 1978.

[18] — On the Algebra of Test Functions for Field Operators. Decomposition of Linear Functionals into Positive Ones, Commun. Math. Phys., 34 (1973), 315-333.

Note added in proof: Problem (1) has been answared in the negative (J. Alcántara: Some new results on topological tensor products, The Open Univ. preprint); and Hofmann has given an answer to (2) (Beschreibung der Extremalstraplen des Positivitätskegels in Tensoralgebren, Leipzig preprint.) 